\title{
An interlaboratory comparison programme on high frequency electromagnetic field measurements in a controllable environment performed in Greece
}

\author{
Eleni Nicolopoulou ${ }^{1}$, Ioannis Ztoupis ${ }^{1}$, Efthymios Karabetsos ${ }^{2 a}$, Ioannis Gonos ${ }^{1}$ and Ioannis Stathopulos ${ }^{1}$ \\ ${ }^{1}$ National Technical University of Athens, High Voltage Laboratory, 15780 Zografou Campus, Greece \\ ${ }^{2}$ Greek Atomic Energy Commission, Non Ionizing Radiation Office, 15310 Agia Paraskevi, Greece
}

\begin{abstract}
An interlaboratory comparison scheme (ILC) on high frequency electromagnetic field measurements has been conducted in order to evaluate the overall performance of laboratories that perform measurements in the vicinity of mobile phone base stations and broadcast antenna facilities. The participants recorded the electric field produced by two high frequency signal generators inside an anechoic chamber in three measurement scenarios with the antennas transmitting each time different signals at the FM, VHF, UHF and GSM frequency bands. In each measurement scenario, the participants also used their measurements to calculate the relative exposure ratios. In this paper, the estimates of the mean and standard deviation of the measurements were defined utilizing an iterative robust algorithm and the participants' results were subsequently evaluated in each test level calculating the performance statistics z-scores. Based on these calculations of the $\mathrm{z}$ scores, possible sources of errors for each participating laboratory were discussed and the overall evaluation of their performance was determined by using an aggregated performance statistic.
\end{abstract}

\section{Introduction}

According to the Greek legislation [1], measurements of the electromagnetic fields levels in the vicinity of mobile phone base stations and broadcast antenna facilities are carried out by accredited laboratories in order to evaluate the exposure levels of the general public. The relevant metric for assessing compliance with exposure limits is the total exposure ratio [2-4]. This is the sum of the individual exposure ratios concerning the same quantity (electric or magnetic field) and the same effect (thermal or electrical stimulation) at a measurement location at a specific time slot. For frequencies between $10 \mathrm{MHz}$ and $10 \mathrm{GHz}$ and for measurements in the far field of the radiating antenna, the exposure ratio (ER) for a specific frequency (or frequency band) and measurement position is calculated as follows:

$$
E R=\frac{E^{2}}{L_{E}^{2}}=\frac{H^{2}}{L_{H}^{2}}
$$

where:

$E$ (or $H$ ) the average value of the measured electric (or magnetic) field at a certain frequency and measurement position

$L_{E}$ (or $L_{H}$ ) the corresponding reference level for the electric (or magnetic) field at this frequency.
According to the standard ISO/IEC 17025 [5], every test laboratory should participate in interlaboratory comparison programmes (ILCs) in order to demonstrate or assess its adequacy and improve its performance. Interlaboratory comparison programmes are defined as the organization, performance and evaluation of calibration/tests on the same or similar calibration/test items by two or more laboratories under predetermined conditions. The type of ILC that must be applied to electromagnetic field measurements is a results comparison programme where all participants measure the same EMF source, usually in normal working conditions [6, 7]. The ILC programme described in this paper has been set up to comply with the requirements of the ILAC G13: 2000 Guidelines [8], the ILAC Policy [9] and the relevant policy of the Hellenic Accreditation System S.A. (ESYD) for the participation of laboratories in proficiency testing schemes [10].

\section{Interlaboratory Comparison Scheme}

An interlaboratory comparison scheme (ILC) was designed, which involved high frequency electromagnetic field measurements and calculations of exposure ratios in different measurement scenarios. The ILC scheme on high frequency electromagnetic field measurements was organised by the Greek Atomic Energy Commission and

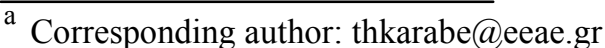


carried out at the High Voltage Laboratory of the National Technical University of Athens. The ILC scheme was conducted in May 2013 and lasted for three days. The purpose of this interlaboratory comparison was threefold:

1) To allow the participating laboratories for objective verification of their technical competence following international standards,

2) To evaluate the overall performance of the participants under totally controlled conditions,

3) To determine the error factors in high frequency electromagnetic field measurements for each participant.

Details about the participating laboratories, the measuring apparatus and the measurement procedure of the ILC scheme are described in the below subsections.

\subsection{Participants}

Nineteen laboratories with thirty groups have participated in this interlaboratory comparison procedure. A unique laboratory identification code number was assigned to each participating group (Laboratories 1-30) and this identity was kept secret, thus guaranteeing data confidentiality. It must be noted that a measurement is defined as the unique combination of personnel handling the instruments, measuring equipment and measurement procedure. A measurement repeated at a specific site with another combination of these factors is considered a different measurement and laboratories that implemented this practice received different codes, one for each measurement group.

\subsection{Measuring equipment}

The participating teams used different types of measuring equipment. Specifically, 25 groups performed the measurements of high frequency electromagnetic fields with hand-held frequency-selective field strength measuring devices (Narda Selective Radiation Meters models 3000, 3006) in combination with isotropic probes. Also, 2 participating groups used a broadband survey field meter (Narda EMR-300) for readings of averaged fields across the whole frequency range of this equipment. However, 3 participating teams (Laboratories 8,15 and 16) used portable spectrum analyzers in conjunction with antennas. Specifically, Lab 8 used the R\&S HE200 antenna (20 MHz - $3 \mathrm{GHz}$ ), Lab 15 the R\&S isotropic antenna $(30 \mathrm{MHz}-3 \mathrm{GHz})$, and $\mathrm{Lab} 16$ the Aaronia/Bicolog 30100 Antenna $(30 \mathrm{MHz}-1 \mathrm{GHz})$ and $\mathrm{Z}$ Technology/Log Periodic AA8 Antenna $(800 \mathrm{MHz}-$ $2,6 \mathrm{GHz})$.

\subsection{Measurement procedure}

The measurements of high frequency fields were conducted in the facilities of the High Voltage Laboratory of the National Technical University of Athens. Specifically, the participating laboratories measured at one predefined position inside the shielded anechoic chamber $(6,4 \mathrm{mX} 3,45 \mathrm{mX} 3,05 \mathrm{~m})$ according to three measurement scenarios.
The measured field within the anechoic chamber was produced by two signal generators which were connected via two power amplifiers with two antennas, respectively. The field was controllable by selecting the characteristics of the generators' waveforms. In each scenario, each combination of signal generator - power amplifier antenna, emitted field at specific frequencies. Also, the first antenna (R\&S HE200) was vertically oriented with respect to the floor and the second one (Teseq CBL6111D BiLog Antenna) was placed at an angle of approximately 45 degrees in order to examine the capacity of the laboratories to detect the three spatial components of the fields. The location and orientation of the antennas are depicted in Figure 1.

Figure 1. Location and orientation of the antennas inside the anechoic chamber.

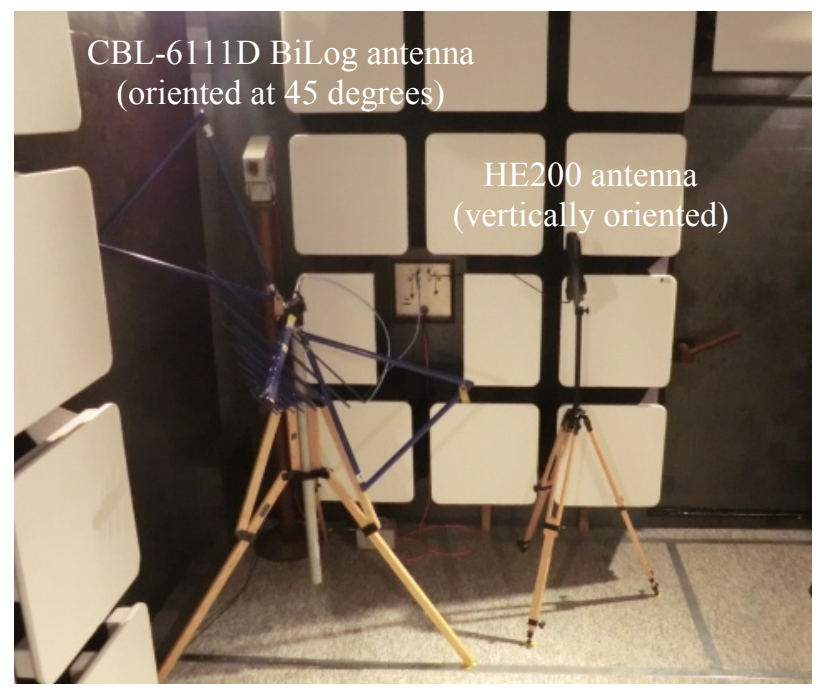

In all scenarios, the position where the measurements were taken was constant at a height of $1.5 \mathrm{~m}$ and about $4 \mathrm{~m}$ away from the axis of the transmitting antennas. Consequently, the measurements were made in the far field of the antennas. The schematic diagram of the experimental setup is shown in the following figure.

Figure 2. Schematic of the exposure setup for the ILC.

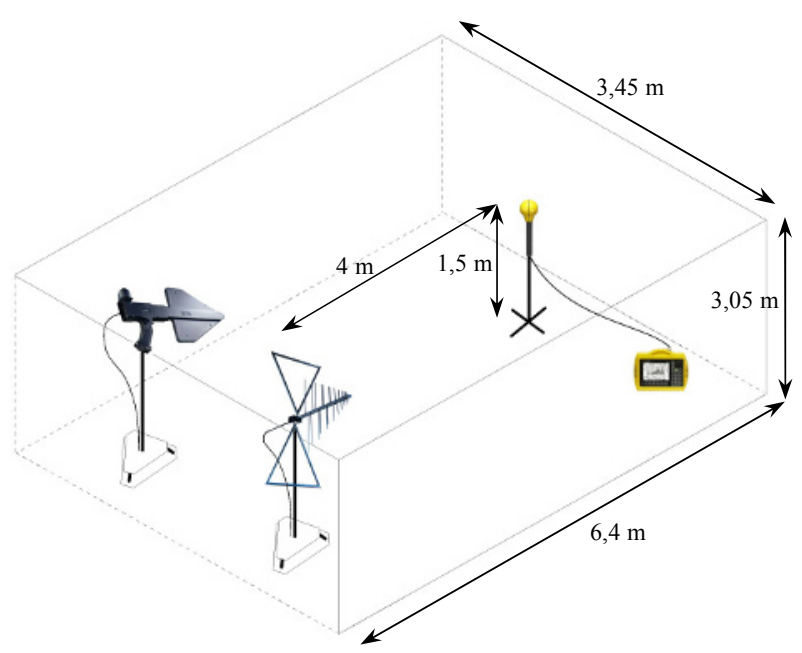


In each of the three scenarios, the broadcast frequencies of each generator used in the interlaboratory comparison are presented in Table 1 below. It should be noted that the laboratories did not know the characteristics of the incident field that they were asked to measure in any of the three scenarios.

Table 1. Broadcast frequencies for the ILC scheme.

\begin{tabular}{|c|c|c|}
\hline & Signal generator 1 & Signal generator 2 \\
\hline $\begin{array}{c}\text { Measurement } \\
\text { scenario 1 }\end{array}$ & $\begin{array}{c}\text { FM } \\
(102,5 \mathrm{MHz})\end{array}$ & $\begin{array}{c}\text { GSM } \\
(935 \mathrm{MHz})\end{array}$ \\
\hline $\begin{array}{c}\text { Measurement } \\
\text { scenario 2 }\end{array}$ & $\begin{array}{c}\mathrm{FM} \\
(96,3 \mathrm{MHz})\end{array}$ & $\begin{array}{c}\mathrm{UHF} \\
(440 \mathrm{MHz})\end{array}$ \\
\hline $\begin{array}{c}\text { Measurement } \\
\text { scenario 3 }\end{array}$ & $\begin{array}{c}\mathrm{VHF} \\
(180 \mathrm{MHz})\end{array}$ & $\begin{array}{c}\mathrm{UHF} \\
(720 \mathrm{MHz})\end{array}$ \\
\hline
\end{tabular}

\subsection{Measurement task}

The laboratories carried out the measurements taking into account the instructions given by the organizer. At each measurement scenario they were asked to measure the following measurands:

- A 6-minute average value of the electric field strength $\left(E_{T O T}\right)$ across the total measured spectral range.

- The maximum values of the electric field strength $\left(E_{F M}, E_{V H F}, E_{U H F}\right.$ and $\left.E_{G S M}\right)$ in the two frequency bands at which they detected the maximum emission level.

For each laboratory, a time slot of thirty minutes has been allocated to execute the measurements. After completing the measurements, the participating teams had to calculate at each measurement scenario:

- The total exposure ratio $\left(E R_{T O T}\right)$ throughout the whole spectral range (applying reduction factor limits $60 \%$, as imposed by the Greek legislation [1]).

- The exposure ratios ( $E R_{F M}, E R_{V H F}, E R_{U H F}$ and $\left.E R_{G S M}\right)$ in both frequency bands with the highest measured value of electric field strength.

All participants had 3 days at their disposal to deliver the 18 quantities (6 for each measurement scenario).

\section{Performance statistics}

\subsection{Calculation of z-scores}

Before evaluating the results a statistical analysis must be carried out in order to determine the best available estimations for the true value of the measurand (assigned value $\widehat{m}$ ) and the dispersion of the measurements (standard deviation for the proficiency assessment $\widehat{\sigma}$ ). In the present scheme these estimators are calculated in each test level by applying the iterative robust algorithm described in the standard ISO 13528 (Annex C, Algorithm A) [12] to the measurements of the laboratories. The robust parameters are then used for the calculation of the performance statistic $\mathrm{z}$ score from the relationship:

$$
z=\frac{x-\hat{m}}{\widehat{\sigma}}
$$

where:

$x$ is the measurement of the laboratory

$\widehat{m}$ is the robust mean

$\hat{\sigma}$ is the robust standard deviation

For the evaluation of the results, the $\mathrm{z}$ scores are divided into the following regions [12]:

- $|z| \leq 2$ : the performance of the laboratory is satisfactory.

- $2<|z|<3$ : the accuracy and correctness of the measurement is questionable and the performance statistic is a "warning signal".

- $|z| \geq 3$ : the performance of the laboratory is nonsatisfactory and the performance statistic is an "action signal".

Each "action signal" is a strong indication of problems in the measuring performance of the laboratory and further investigation is required for the determination of the error factors which affect the measurement quality. "Warning signals" are milder indicators of inaccuracy sources and raise concerns mainly if they reappear in various test levels (or various test rounds).

\subsection{Acceptance criterion set by the organizer}

If the measurements follow the normal distribution and the values $\hat{m}$ and $\hat{\sigma}$ are good estimators, then the $\mathrm{z}$ score is a normally distributed random variable, with mean value 0 and standard deviation 1 , regardless of the measurand, the test level and the measurement method. This enables comparing the individual $\mathrm{z}$ scores and combining them to create an aggregated score for the whole scheme round which provides every participant with a simple overview of its performance in all test levels. The type of aggregated score, which has been chosen in this ILC scheme, is the number of individual test levels, where the participant has been evaluated with a $|z|>2$.

As it is known, a variable described by the normal distribution $\mathrm{N}\left(\mu, \sigma^{2}\right)$ lies with approximately $95 \%$ probability in the interval $\mu \pm 2 \sigma$ and with $99,7 \%$ probability in the interval $\mu \pm 3 \sigma$. Thus, the $z$ scores of a laboratory- which follow the distribution $\mathrm{N}(0,1)$ - are expected to lie outside the region \pm 2 in $5 \%$ of the measurements and outside the region \pm 3 only for $0,3 \%$ of the measurements. A well behaving laboratory is expected to comply with these limits.

Therefore, by calculating for each participant the number of individual test levels where it has received a $|z|>2$ as \% percentage of the total number of its evaluated measurements, the following acceptance criterion arises:

- If this percentage exceeds 5\%, the overall performance of the laboratory is non-satisfactory.

- If this percentage does not exceed $5 \%$, the overall performance of the laboratory is satisfactory. 


\subsection{Results}

The $\mathrm{z}$ scores referring to the measurements of the electric field strength and the calculation of the exposure ratios reported by the participants are analytically presented in Table 2. The total number of $|z|>2$ and the resulting \% percentage are also presented. Cells with non-satisfactory results are highlighted. The aggregated performance statistics for the overall evaluation of the high frequency ILC scheme are shown graphically in Figure 2.

Figure 2. Evaluation of the participants: percentage of test levels where $|z|>2$ for each laboratory.

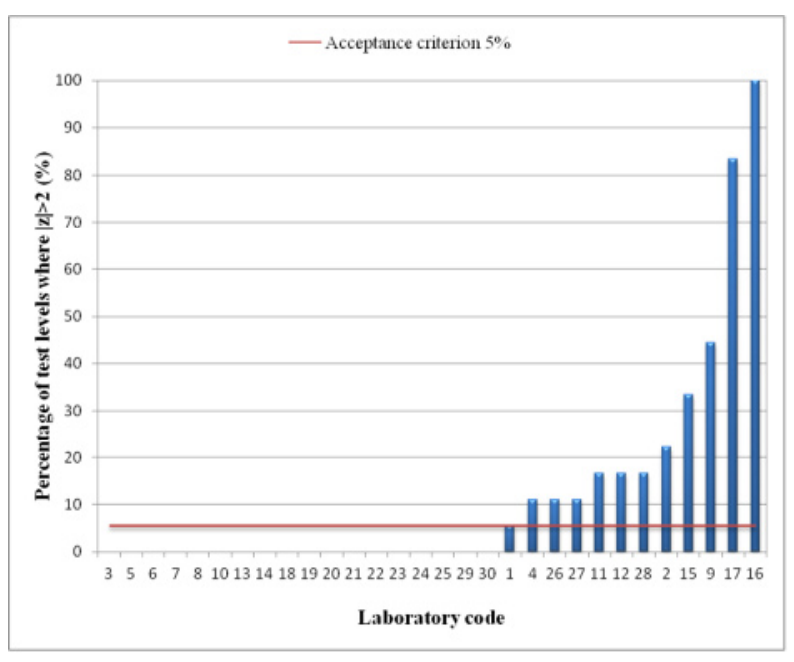

\section{Discussion - Conclusions}

The purpose of the presented work was to describe the organisation and execution of an ILC scheme on high frequency electromagnetic field measurements and to assess its overall function. The analysis of the results provides useful conclusions about possible error sources within the laboratories:

Instrumentation in general and inappropriate settings used in the measuring equipment are the major factors that affect the accuracy of the results. In the present ILC scheme, 15 out of the 25 teams that performed frequencyselective measurements with the Narda Selective Radiation Meters (SRM) had an overall satisfying performance. On the contrary, 2 out of the 3 teams that used a spectrum analyzer were negatively evaluated (Laboratories 15 and 16).

The adjustment of the settings, the implementation of the measurement procedure and the processing of the results should be done with special precaution when using a spectrum analyzer. Especially the use of a nonisotropic antenna in conjunction with the spectrum analyzer should be followed by a proper combination of the 3 spatial field components. The use of a non-isotropic antenna has yielded worse results for Lab 16 compared to the isotropic antenna of Lab 15.

Apart from the polarization of the field, the frequency range of the antenna used is an important factor. In measurement scenario 1, which included a maximum emission in the FM band $(102,5 \mathrm{MHz})$, some of the action signals Lab 16 has received are attributed to the operating frequency range $(800-2600 \mathrm{MHz})$ of the used Log Periodic antenna.

The majority of the participants conducted measurements that covered the frequency range $75 \mathrm{MHz}-$ $3 \mathrm{GHz}$. Laboratories 2 and 17 that reported a wider total frequency range $(27 \mathrm{MHz}-3 \mathrm{GHz})$ recorded higher field values -possibly because they detected harmonics emitted by the generators- and received action signals for their electric field measurements.

An additional very important technical characteristic related to the spectral sensitivity of the measuring equipment is the Resolution Bandwidth (RBW). Improper adjustment of this parameter explains the action signals of Laboratory 9. The RBW settings for measurement scenario $1(2 \mathrm{kHz}$ for the FM band, $30 \mathrm{kHz}$ for the GSM band, whereas the other participants used $100 \mathrm{kHz}$ and $200 \mathrm{kHz}$ respectively) resulted in an $E_{F M}$ value significantly higher than the total field $E_{T O T}$. Similarly, in measurement scenario 2, the RBW in the FM band was retained at $2 \mathrm{kHz}$ and in the UHF band it was set at $200 \mathrm{kHz}$, much smaller than the setting used by the other teams $(1 \mathrm{MHz})$. Moreover, the measurement range of the instrument in measurement scenario 1 was set at $2,8 \mathrm{~V} / \mathrm{m}$, a value much smaller than the recorded field values, which are therefore considered to have questionable accuracy.

Laboratories 11 and 12 (which had the same operators but different measuring equipment, SRM 3000 and SRM 3006 respectively) received action signals, in all three scenarios, only for the total exposure ratios. This indicates an error during the processing of the measurements or the application of the exposure limits for the calculation of the exposure ratio.

Non repetitive $|z|>2$ without obvious error sources as in the cases of Laboratories 1, 2, 26, 27 and 28 could be treated as occasional mistakes. It should be pointed out that a measurement of the electric field evaluated with a $|z|>2$ is very likely to cause a $|z|>2$ in the corresponding exposure ratio.

The execution of the scheme in a controllable environment such as an anechoic chamber with predefined emission conditions aimed at assuring maximum comparability of the measurements by eliminating the temporal variation of the field sources. The detection of possible technical weaknesses in most cases proves the satisfying function of the scheme. The fact that over one third of the participants (12 out of 30 measuring teams) had a non satisfying performance shows that technical competence on high frequency EMF measurements needs to be constantly recorded and evaluated through participation in ILC schemes.

\section{References}

1. Law No 4070, Act. No. 82/A/10.04.2012: "Regulations concerning electronic communications, transport, public works and other provisions"

2. ELOT 1422-3 : "Radiocommunication Antenna collocation - Part 3: Testing and measurement techniques - Limits" 
3. CEPT Revised ECC/REC/(02)04, "Measuring nonionising electromagnetic radiation $(9 \mathrm{kHz}-300 \mathrm{GHz})$ ",

4. EN 50492: 2008, "Basic standard for the in-situ measurement of electromagnetic field strength related to human exposure in the vicinity of base stations"

5. ISO/ IEC 17025: 2005, "General requirements for the competence of testing and calibration laboratories"

6. P. Bienkowski, H. Trzaska, "Interlaboratory comparisons in EMF survey measurements - Methods and results", Proceedings of International conference and COST 281 Workshop on Emerging EMF Technologies, Potential Sensitive Groups and Health, Graz, April 20-21 (2006)

7. P. Bienkowski, "Interlaboratory comparisons in EMF survey measurements", Environmentalist, 29, 130134 (2009)

8. ILAC G13: 2000, "Guidelines for the requirements for the competence of providers of proficiency testing schemes"

9. ILAC-P9:2005, "ILAC Policy for Participation in National and International Proficiency Testing Activities" 10. ESYD PDI/02/01/02-09-2011: "ESYD policy relevant to PT Schemes and Interlaboratory Comparisons" http://www.esyd.gr/pweb/s/20/files/EN/ kanonismoi/ PDI_27_1_11.pdf)

11. ISO 13528: 2005 , "Statistical Methods for Use in Proficiency Testing by Interlaboratory Comparisons" 


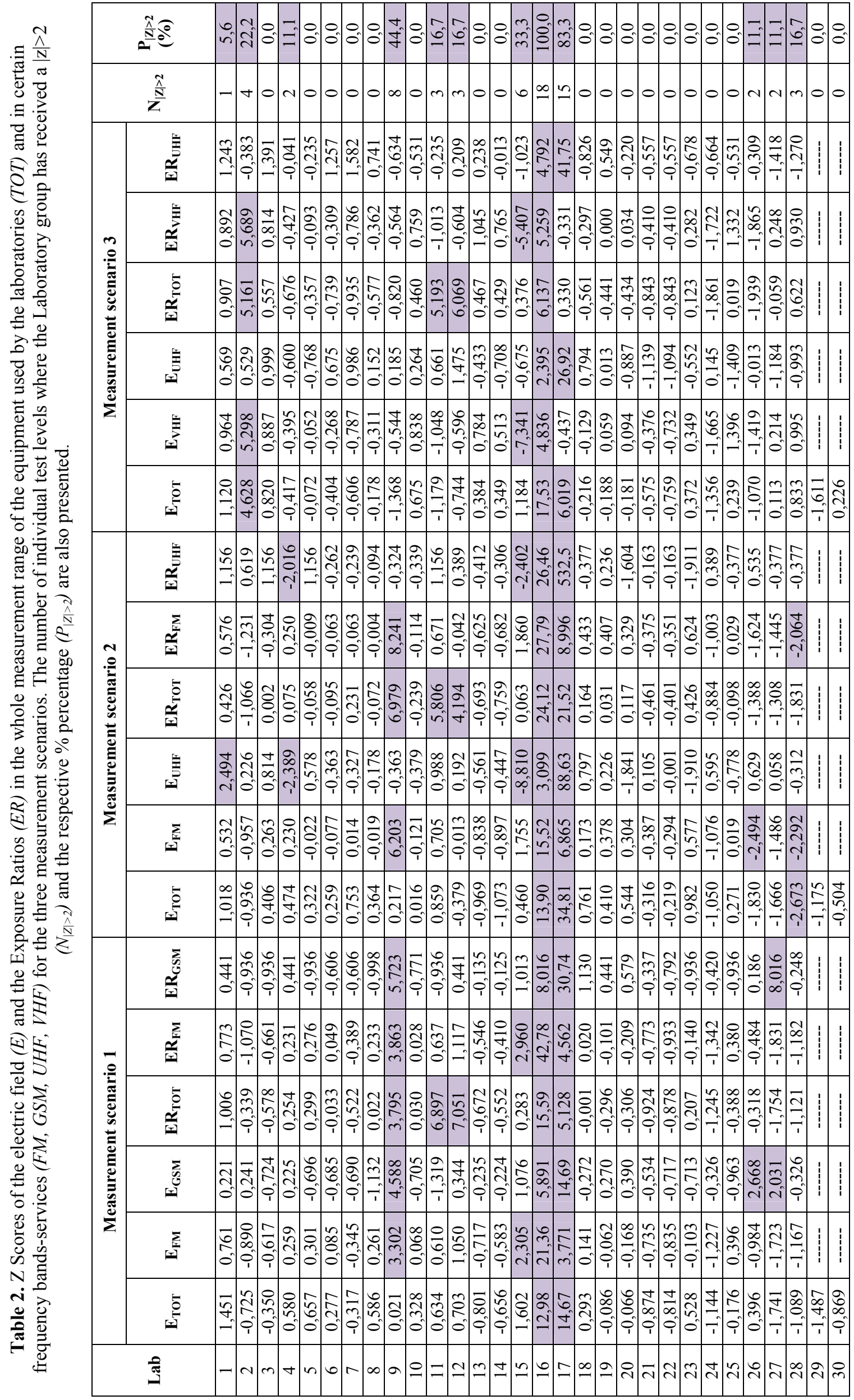

